\title{
Definition of Flat Poset and Existence Theorems for Recursive Call
}

\author{
Kazuhisa Ishida \\ Neyagawa-shi \\ Osaka, Japan
}

\author{
Yasunari Shidama ${ }^{1}$ \\ Shinshu University \\ Nagano, Japan
}

\author{
Adam Grabowski \\ Institute of Informatics \\ University of Białystok \\ Akademicka 2, 15-267 Białystok \\ Poland
}

\begin{abstract}
Summary. This text includes the definition and basic notions of product of posets, chain-complete and flat posets, flattening operation, and the existence theorems of recursive call using the flattening operator. First part of the article, devoted to product and flat posets has a purely mathematical quality. Definition 3 allows to construct a flat poset from arbitrary non-empty set 12 in order to provide formal apparatus which eanbles to work with recursive calls within the Mizar langauge. To achieve this we extensively use technical Mizar functors like BaseFunc or RecFunc. The remaining part builds the background for information engineering approach for lists, namely recursive call for posets 21. We formalized some facts from Chapter 8 of this book as an introduction to the next two sections where we concentrate on binary product of posets rather than on a more general case.
\end{abstract}

MSC: 06A11 68N30 03B35

Keywords: flat posets; recursive calls for posets; flattening operator

MML identifier: POSET_2, version: 8.1.02 5.22.1199

The notation and terminology used in this paper have been introduced in the following articles: [1], [17, [1], 6], [7, 8], 2], 13], 19], 14], 4], 9], 15, [22], [23], 20], [5], [16], and [10].

\footnotetext{
${ }^{1}$ My work was supported by JSPS KAKENHI 22300285.
}

(C) 2014 University of Białystok
CC-BY-SA License ver. 3.0 or later ISSN 1426-2630(Print), 1898-9934(Online) 


\section{Preliminaries from Poset Theory}

From now on $a, Z_{1}, Z_{2}, Z_{3}$ denote sets, $x, y, z$ denote objects, and $k$ denotes a natural number.

Now we state the propositions:

(1) Let us consider a lower-bounded non empty poset $P$ and an element $p$ of $P$. If $p \leqslant$ the carrier of $P$, then $p=\perp_{P}$.

(2) Let us consider a chain-complete non empty poset $P$, a non empty chain $L$ of $P$, and an element $p$ of $P$. If $p \in L$, then $p \leqslant \sup L$.

(3) Let us consider a chain-complete non empty poset $P$, a non empty chain $L$ of $P$, and an element $p_{1}$ of $P$. Suppose an element $p$ of $P$. If $p \in L$, then $p \leqslant p_{1}$. Then $\sup L \leqslant p_{1}$.

\section{On the Product of Posets}

Now we state the proposition:

(4) Let us consider non empty relational structures $P, Q$ and an object $x$. Then $x$ is an element of $P \times Q$ if and only if there exists an element $p$ of $P$ and there exists an element $q$ of $Q$ such that $x=\langle p, q\rangle$.

Let $P, Q$ be non empty posets and $L$ be a non empty chain of $P \times Q$. The functors: $\pi_{1}(L)$ and $\pi_{2}(L)$ yield non empty chains of $P$. Let $P, Q_{1}, Q_{2}$ be non

empty posets, $f_{1}$ be a monotone function from $P$ into $Q_{1}$, and $f_{2}$ be a monotone function from $P$ into $Q_{2}$. One can verify that $\left\langle f_{1}, f_{2}\right\rangle$ is monotone as a function from $P$ into $Q_{1} \times Q_{2}$.

Let $P, Q$ be chain-complete non empty posets. Observe that $P \times Q$ is chaincomplete.

Now we state the proposition:

(5) Let us consider chain-complete non empty posets $P, Q$ and a non empty chain $L$ of $P \times Q$. Then $\sup L=\left\langle\sup \pi_{1}(L), \sup \pi_{2}(L)\right\rangle$.

Let $P, Q_{1}, Q_{2}$ be strict chain-complete non empty posets, $f_{1}$ be a continuous function from $P$ into $Q_{1}$, and $f_{2}$ be a continuous function from $P$ into $Q_{2}$. Note that $\left\langle f_{1}, f_{2}\right\rangle$ is continuous as a function from $P$ into $Q_{1} \times Q_{2}$. 


\section{Definition of Flat Poset and Poset Flattening}

Let $I_{3}$ be a relational structure. We say that $I_{3}$ is flat if and only if

(Def. 1) There exists an element $a$ of $I_{3}$ such that for every elements $x, y$ of $I_{3}$, $x \leqslant y$ iff $x=a$ or $x=y$.

One can verify that every non empty relational structure which is discrete is also reflexive and every discrete non empty relational structure which is trivial is also flat and there exists a poset which is strict, non empty, and flat and every relational structure which is flat is also reflexive transitive and antisymmetric and every non empty poset which is flat is also lower-bounded.

In the sequel $S$ denotes a relational structure, $P, Q$ denote non empty flat posets, $p, p_{1}, p_{2}$ denote elements of $P$, and $K$ denotes a non empty chain of $P$.

Now we state the proposition:

(6) Let us consider a non empty flat poset $P$ and a non empty chain $K$ of $P$. Then there exists an element $a$ of $P$ such that $K=\{a\}$ or $K=\left\{\perp_{P}, a\right\}$.

Let us consider a function $f$ from $P$ into $Q$. Now we state the propositions:

(7) There exists an element $a$ of $P$ such that $K=\{a\}$ and $f^{\circ} K=\{f(a)\}$ or $K=\left\{\perp_{P}, a\right\}$ and $f^{\circ} K=\left\{f\left(\perp_{P}\right), f(a)\right\}$. The theorem is a consequence of $(6)$.

(8) If $f\left(\perp_{P}\right)=\perp_{Q}$, then $f$ is monotone.

Now we state the proposition:

(9) If $K=\left\{\perp_{P}, p\right\}$, then $\sup K=p$.

One can verify that there exists a poset which is strict, non empty, flat, and chain-complete and every poset which is non empty and flat is also chaincomplete.

Now we state the proposition:

(10) Let us consider strict non empty chain-complete flat posets $P, Q$ and a function $f$ from $P$ into $Q$. If $f\left(\perp_{P}\right)=\perp_{Q}$, then $f$ is continuous. Proof: For every non empty chain $K$ of $P, f(\sup K) \leqslant \sup \left(f^{\circ} K\right)$ by [15, (1)], (7), [5, (39)], (9).

\section{Primaries for Existence Theorems of Recursive Call Using FLATTENING}

In the sequel $X, Y$ denote non empty sets.

Let $X$ be a non empty set. The functor FlatRelat $X$ yielding a relation between succ $X$ and succ $X$ is defined by the term

(Def. 2) $(\{\langle X, X\rangle\} \cup\{X\} \times X) \cup \operatorname{id}_{X}$.

Now we state the proposition: 
(11) Let us consider elements $x, y$ of $\operatorname{succ} X$. Then $\langle x, y\rangle \in$ FlatRelat $X$ if and only if $x=X$ or $x=y$.

Let $X$ be a non empty set. The functor FlatPoset $X$ yielding a strict non empty chain-complete flat poset is defined by the term

(Def. 3) $\langle$ succ $X$, FlatRelat $X\rangle$.

Now we state the propositions:

(12) Let us consider elements $x, y$ of FlatPoset $X$. Then $x \leqslant y$ if and only if $x=X$ or $x=y$.

(13) $X$ is an element of FlatPoset $X$.

Let us consider $X$. Let us observe that $\perp_{\text {FlatPoset } X}$ reduces to $X$.

Let $x$ be an object, $X, Y$ be non empty sets, and $f$ be a function from $X$ into $Y$. The functor Flatten $(f, x)$ yielding a set is defined by the term

$$
\begin{cases}f(x), & \text { if } x \in X \\ Y, & \text { otherwise. }\end{cases}
$$

The functor Flatten $(f)$ yielding a function from FlatPoset $X$ into FlatPoset $Y$ is defined by

(Def. 5) (i) $i t(X)=Y$, and

(ii) for every element $x$ of FlatPoset $X$ such that $x \neq X$ holds it $(x)=$ $f(x)$.

Let us observe that Flatten $(f)$ is continuous.

Now we state the proposition:

(14) Let us consider a function $f$ from $X$ into $Y$.

If $x \in X$, then $($ Flatten $(f))(x) \in Y$.

Let us consider $X$ and $Y$. The functor $\operatorname{FlatConF}(X, Y)$ yielding a strict chain-complete non empty poset is defined by the term

(Def. 6) ConPoset(FlatPoset $X$, FlatPoset $Y$ ).

Let $L$ be a flat poset. One can verify that every chain of $L$ is finite and there exists a lattice which is non empty, flat, and lower-bounded.

Now we state the propositions:

(15) Let us consider a non empty lattice $L$, an element $x$ of $L$, and an $x$-chain $A$ of $x$. Then $\overline{\bar{A}}=1$. Proof: For every element $z$ of $L$ such that $z \in A$ holds $z \in\{x\}$ by [19, (2)].

(16) Let us consider a non empty flat lower-bounded lattice $L$, an element $x$ of $L$, and a $\perp_{L}$-chain $A$ of $x$. Then $\overline{\bar{A}} \leqslant 2$. The theorem is a consequence of (6) and (15).

(17) Let us consider a finite lower-bounded antisymmetric non empty lattice $L$. Then $L$ is flat if and only if for every element $x$ of $L$, height $x \leqslant 2$. Proof: There exists an element $a$ of $L$ such that for every elements $x, y$ of $L, x \leqslant y$ iff $x=a$ or $x=y$ by [5, (44)], [13, (2), (6)], [3, (13)]. 


\section{Existence Theorem of Recursive Call for Single-equation}

From now on $D$ denotes a subset of $X, I$ denotes a function from $X$ into $Y, J$ denotes a function from $X \times Y$ into $Y$, and $E$ denotes a function from $X$ into $X$.

Let $X$ be a non empty set, $D$ be a subset of $X$, and $E$ be a function from $X$ into $X$. We say that $E$ is well founded with minimal set $D$ if and only if

(Def. 7) There exists a function $l$ from $X$ into $\mathbb{N}$ such that for every element $x$ of $X$, if $l(x) \leqslant 0$, then $x \in D$ and if $x \notin D$, then $l(E(x))<l(x)$.

Let $X, Y$ be non empty sets. Let $I$ be a function from $X$ into $Y, J$ be a function from $X \times Y$ into $Y$, and $x, y$ be objects. The functor BaseFunc01 $(x, y, I, J, D)$ yielding a set is defined by the term

(Def. 8) $\begin{cases}I(x), & \text { if } x \in D, \\ J(\langle x, y\rangle), & \text { if } x \notin D \text { and } x \in X \text { and } y \in Y, \\ Y, & \text { otherwise. }\end{cases}$

Let $E$ be a function from $X$ into $X$ and $h$ be an object. Assume $h$ is a continuous function from FlatPoset $X$ into FlatPoset $Y$.

The functor RecFunc01( $h, E, I, J, D)$ yielding a continuous function from FlatPoset $X$ into FlatPoset $Y$ is defined by

(Def. 9) Let us consider an element $x$ of FlatPoset $X$ and a continuous function $f$ from FlatPoset $X$ into FlatPoset $Y$. Suppose $h=f$. Then it $(x)=$ BaseFunc01 $(x, f(($ Flatten $(E))(x)), I, J, D)$.

Now we state the propositions:

(18) There exists a continuous function $W$ from $\operatorname{FlatConF}(X, Y)$ into FlatConF $(X, Y)$ such that for every element $f$ of ConFuncs(FlatPoset $X$, FlatPoset $Y$ ), $W(f)=\operatorname{RecFunc01}(f, E, I, J, D)$. Proof: Set $F_{1}=$ FlatPoset $X$. Set $F_{2}=$ FlatPoset $Y$. Set $F_{3}=$ FlatConF $(X, Y)$. Set $C_{1}=\operatorname{ConFuncs}\left(F_{1}, F_{2}\right)$. Define $\mathcal{H}$ (object) $=\operatorname{RecFunc01}\left(\$_{1}, E, I, J, D\right)$. For every continuous function $h$ from $F_{1}$ into $F_{2}, h \in C_{1}$ by [7, (8)]. For every set $h$ such that $h \in C_{1}$ holds $h$ is a continuous function from $F_{1}$ into $F_{2}$. There exists a function $W$ from $C_{1}$ into $C_{1}$ such that for every object $f$ such that $f \in C_{1}$ holds $W(f)=\mathcal{H}(f)$ from [7, Sch. 2]. Consider $I_{3}$ being a function from $C_{1}$ into $C_{1}$ such that for every object $f$ such that $f \in C_{1}$ holds $I_{3}(f)=\mathcal{H}(f) . I_{3}$ is a continuous function from $F_{3}$ into $F_{3}$ by [7, (5)], (12), [24, (9)], [15, (1), (11)].

(19) There exists a set $f$ such that

(i) $f \in$ ConFuncs(FlatPoset $X$, FlatPoset $Y$ ), and

(ii) $f=\operatorname{RecFunc01}(f, E, I, J, D)$.

The theorem is a consequence of (18). 
Let us assume that $E$ is well founded with minimal set $D$. Now we state the propositions:

(20) There exists a continuous function $f$ from FlatPoset $X$ into FlatPoset $Y$ such that for every element $x$ of $X, f(x) \in Y$ and $f(x)=\operatorname{BaseFunc01(x,f}$ $(E(x)), I, J, D)$. Proof: Consider $f$ being a set such that $f \in$ ConFuncs (FlatPoset $X$, FlatPoset $Y$ ) and $f=\operatorname{RecFunc01}(f, E, I, J, D)$. Consider $l$ being a function from $X$ into $\mathbb{N}$ such that for every element $x_{0}$ of $X$, if $l\left(x_{0}\right) \leqslant 0$, then $x_{0} \in D$ and if $x_{0} \notin D$, then $l\left(E\left(x_{0}\right)\right)<l\left(x_{0}\right)$. Define $\mathcal{P}$ [natural number] $\equiv$ for every element $x_{0}$ of $X$ such that $l\left(x_{0}\right) \leqslant \$_{1}$ holds $f\left(x_{0}\right) \in Y$ and $f\left(x_{0}\right)=$ BaseFunc01 $\left(x_{0}, f\left(E\left(x_{0}\right)\right), I, J, D\right) . \mathcal{P}[0]$ by [7. (5)]. For every $k$ such that $\mathcal{P}[k]$ holds $\mathcal{P}[k+1]$ by [7, (5)], [3, (13)]. For every natural number $k, \mathcal{P}[k]$ from [3, Sch. 2]. For every element $x$ of $X$, $f(x) \in Y$ and $f(x)=\operatorname{BaseFunc01}(x, f(E(x)), I, J, D)$.

(21) There exists a function $f$ from $X$ into $Y$ such that for every element $x$ of $X$, if $x \in D$, then $f(x)=I(x)$ and if $x \notin D$, then $f(x)=J(\langle x, f(E(x))\rangle)$. Now we state the proposition:

(22) Let us consider functions $f_{1}, f_{2}$ from $X$ into $Y$. Suppose

(i) $E$ is well founded with minimal set $D$, and

(ii) for every element $x$ of $X$, if $x \in D$, then $f_{1}(x)=I(x)$ and if $x \notin D$, then $f_{1}(x)=J\left(\left\langle x, f_{1}(E(x))\right\rangle\right)$, and

(iii) for every element $x$ of $X$, if $x \in D$, then $f_{2}(x)=I(x)$ and if $x \notin D$, then $f_{2}(x)=J\left(\left\langle x, f_{2}(E(x))\right\rangle\right)$.

Then $f_{1}=f_{2}$. Proof: Consider $l$ being a function from $X$ into $\mathbb{N}$ such that for every element $x$ of $X$, if $l(x) \leqslant 0$, then $x \in D$ and if $x \notin D$, then $l(E(x))<l(x)$. Define $\mathcal{P}$ [natural number] $\equiv$ for every element $x$ of $X$ such that $l(x) \leqslant \$_{1}$ holds $f_{1}(x)=f_{2}(x)$. $\mathcal{P}[0]$. For every $k$ such that $\mathcal{P}[k]$ holds $\mathcal{P}[k+1]$ by [3, (13)]. For every natural number $k, \mathcal{P}[k]$ from [3, Sch. 2]. For every element $x$ of $X, f_{1}(x)=f_{2}(x)$.

\section{Existence Theorem of Recursive Calls for 2-Equations}

From now on $D$ denotes a subset of $X, I, I_{1}, I_{2}$ denote functions from $X$ into $Y, J, J_{1}, J_{2}$ denote functions from $X \times Y \times Y$ into $Y$, and $E_{1}, E_{2}$ denote functions from $X$ into $X$.

Let $X$ be a non empty set, $D$ be a subset of $X$, and $E_{1}, E_{2}$ be functions from $X$ into $X$. We say that $\left(E_{1}, E_{2}\right)$ is well founded with minimal set $D$ if and only if

(Def. 10) There exists a function $l$ from $X$ into $\mathbb{N}$ such that for every element $x$ of $X$, if $l(x) \leqslant 0$, then $x \in D$ and if $x \notin D$, then $l\left(E_{1}(x)\right)<l(x)$ and $l\left(E_{2}(x)\right)<l(x)$. 
Let $X, Y$ be non empty sets. Let $I$ be a function from $X$ into $Y, J$ be a function from $X \times Y \times Y$ into $Y$, and $x, y_{1}, y_{2}$ be objects. The functor BaseFunc02 $\left(x, y_{1}, y_{2}, I, J, D\right)$ yielding a set is defined by the term

(Def. 11) $\begin{cases}I(x), & \text { if } x \in D, \\ J\left(\left\langle x, y_{1}, y_{2}\right\rangle\right), & \text { if } x \notin D \text { and } x \in X \text { and } \mathrm{y}_{1}, y_{2} \in Y, \\ Y, & \text { otherwise. }\end{cases}$

Let $E_{1}, E_{2}$ be functions from $X$ into $X$ and $h_{1}, h_{2}$ be objects. Assume $h_{1}$ is a continuous function from FlatPoset $X$ into FlatPoset $Y$ and $h_{2}$ is a continuous function from FlatPoset $X$ into FlatPoset $Y$. The functor RecFunc02 $\left(h_{1}, h_{2}, E_{1}\right.$, $\left.E_{2}, I, J, D\right)$ yielding a continuous function from FlatPoset $X$ into FlatPoset $Y$ is defined by

(Def. 12) Let us consider an element $x$ of FlatPoset $X$ and continuous functions $f_{1}, f_{2}$ from FlatPoset $X$ into FlatPoset $Y$. Suppose

(i) $h_{1}=f_{1}$, and

(ii) $h_{2}=f_{2}$

Then $i t(x)=$

BaseFunc02 $\left(x, f_{1}\left(\left(\operatorname{Flatten}\left(E_{1}\right)\right)(x)\right), f_{2}\left(\left(\right.\right.\right.$ Flatten $\left.\left.\left.\left(E_{2}\right)\right)(x)\right), I, J, D\right)$.

Now we state the propositions:

(23) There exists a continuous function $W$ from $\operatorname{FlatConF}(X, Y) \times$ FlatConF $(X, Y)$ into FlatConF $(X, Y)$ such that for every set $f$ such that $f \in$ ConFuncs(FlatPoset $X$, FlatPoset $Y$ ) $\times$ ConFuncs(FlatPoset $X$, FlatPoset $Y$ ) holds $W(f)=\operatorname{RecFunc02}\left(f_{\mathbf{1}}, f_{\mathbf{2}}, E_{1}, E_{2}, I, J, D\right)$. Proof: Set $F_{1}=$ FlatPoset $X$. Set $F_{2}=$ FlatPoset $Y$. Set $F_{3}=\operatorname{FlatConF}(X, Y)$. Set $C_{1}=$ $\operatorname{ConFuncs}\left(F_{1}, F_{2}\right)$. Set $F_{4}=F_{3} \times F_{3}$. Set $C_{2}=C_{1} \times C_{1}$. Define $\mathcal{H}$ (object) $=$ RecFunc02 $\left(\$_{11}, \$_{12}, E_{1}, E_{2}, I, J, D\right)$. For every continuous function $h$ from $F_{1}$ into $F_{2}, h \in C_{1}$ by [7, (8)]. For every set $h$ such that $h \in C_{1}$ holds $h$ is a continuous function from $F_{1}$ into $F_{2}$. For every element $h$ of $F_{4}$, there exist continuous functions $h_{1}, h_{2}$ from $F_{1}$ into $F_{2}$ such that $h=\left\langle h_{1}, h_{2}\right\rangle$. There exists a function $W$ from $C_{2}$ into $C_{1}$ such that for every object $f$ such that $f \in C_{2}$ holds $W(f)=\mathcal{H}(f)$ from [7, Sch. 2]. Consider $I_{3}$ being a function from $C_{2}$ into $C_{1}$ such that for every object $f$ such that $f \in C_{2}$ holds $I_{3}(f)=\mathcal{H}(f) . I_{3}$ is a continuous function from $F_{4}$ into $F_{3}$ by [7, (5)], [16, (12)], (12), [24, (9)].

(24) There exist sets $f, g$ such that

(i) $f, g \in$ ConFuncs(FlatPoset $X$, FlatPoset $Y$ ), and

(ii) $f=\operatorname{RecFunc02}\left(f, g, E_{1}, E_{2}, I_{1}, J_{1}, D\right)$, and

(iii) $g=\operatorname{RecFunc02}\left(f, g, E_{1}, E_{2}, I_{2}, J_{2}, D\right)$.

The theorem is a consequence of (23) and (4). 
Let us assume that $\left(E_{1}, E_{2}\right)$ is well founded with minimal set $D$. Now we state the propositions:

(25) There exist continuous functions $f, g$ from FlatPoset $X$ into FlatPoset $Y$ such that for every element $x$ of $X, f(x) \in Y$ and $f(x)=\operatorname{BaseFunc02}(x$, $\left.f\left(E_{1}(x)\right), g\left(E_{2}(x)\right), I_{1}, J_{1}, D\right)$ and $g(x) \in Y$ and $g(x)=\operatorname{BaseFunc02}(x$, $\left.f\left(E_{1}(x)\right), g\left(E_{2}(x)\right), I_{2}, J_{2}, D\right)$. Proof: Consider $f, g$ being sets such that $f, g \in$ ConFuncs(FlatPoset $X$, FlatPoset $Y$ ) and $f=\operatorname{RecFunc02}\left(f, g, E_{1}\right.$,

$\left.E_{2}, I_{1}, J_{1}, D\right)$ and $g=\operatorname{RecFunc02}\left(f, g, E_{1}, E_{2}, I_{2}, J_{2}, D\right)$. Consider $l$ being a function from $X$ into $\mathbb{N}$ such that for every element $x_{0}$ of $X$, if $l\left(x_{0}\right) \leqslant 0$, then $x_{0} \in D$ and if $x_{0} \notin D$, then $l\left(E_{1}\left(x_{0}\right)\right)<l\left(x_{0}\right)$ and $l\left(E_{2}\left(x_{0}\right)\right)<l\left(x_{0}\right)$. Define $\mathcal{P}$ [natural number] $\equiv$ for every elements $x_{1}, x_{2}$ of $X$ such that $l\left(x_{1}\right) \leqslant \$_{1}$ and $l\left(x_{2}\right) \leqslant \$_{1}$ holds $f\left(x_{1}\right) \in Y$ and $f\left(x_{1}\right)=\operatorname{BaseFunc02}\left(x_{1}\right.$, $\left.f\left(E_{1}\left(x_{1}\right)\right), g\left(E_{2}\left(x_{1}\right)\right), I_{1}, J_{1}, D\right)$ and $g\left(x_{2}\right) \in Y$ and $g\left(x_{2}\right)=\operatorname{BaseFunc02}\left(x_{2}\right.$, $\left.f\left(E_{1}\left(x_{2}\right)\right), g\left(E_{2}\left(x_{2}\right)\right), I_{2}, J_{2}, D\right) . \mathcal{P}[0]$ by [7, (5)]. For every $k$ such that $\mathcal{P}[k]$ holds $\mathcal{P}[k+1]$ by [7, (5)], [3, (13)], [18, (69)]. For every natural number $k, \mathcal{P}[k]$ from [3, Sch. 2]. For every elements $x_{1}, x_{2}$ of $X, f\left(x_{1}\right) \in Y$ and $f\left(x_{1}\right)=$ BaseFunc02 $\left(x_{1}, f\left(E_{1}\left(x_{1}\right)\right), g\left(E_{2}\left(x_{1}\right)\right), I_{1}, J_{1}, D\right)$ and $g\left(x_{2}\right) \in Y$ and $g\left(x_{2}\right)=$ BaseFunc02 $\left(x_{2}, f\left(E_{1}\left(x_{2}\right)\right), g\left(E_{2}\left(x_{2}\right)\right), I_{2}, J_{2}, D\right)$ by [3, (11)].

(26) There exist functions $f, g$ from $X$ into $Y$ such that for every element $x$ of $X$, if $x \in D$, then $f(x)=I_{1}(x)$ and $g(x)=I_{2}(x)$ and if $x \notin D$, then $f(x)=$ $J_{1}\left(\left\langle x, f\left(E_{1}(x)\right), g\left(E_{2}(x)\right)\right\rangle\right)$ and $g(x)=J_{2}\left(\left\langle x, f\left(E_{1}(x)\right), g\left(E_{2}(x)\right)\right\rangle\right)$.

Now we state the propositions:

(27) Let us consider functions $f_{1}, g_{1}, f_{2}, g_{2}$ from $X$ into $Y$. Suppose

(i) $\left(E_{1}, E_{2}\right)$ is well founded with minimal set $D$, and

(ii) for every element $x$ of $X$, if $x \in D$, then $f_{1}(x)=I_{1}(x)$ and $g_{1}(x)=$ $I_{2}(x)$ and if $x \notin D$, then $f_{1}(x)=J_{1}\left(\left\langle x, f_{1}\left(E_{1}(x)\right), g_{1}\left(E_{2}(x)\right)\right\rangle\right)$ and $g_{1}(x)=J_{2}\left(\left\langle x, f_{1}\left(E_{1}(x)\right), g_{1}\left(E_{2}(x)\right)\right\rangle\right)$, and

(iii) for every element $x$ of $X$, if $x \in D$, then $f_{2}(x)=I_{1}(x)$ and $g_{2}(x)=$ $I_{2}(x)$ and if $x \notin D$, then $f_{2}(x)=J_{1}\left(\left\langle x, f_{2}\left(E_{1}(x)\right), g_{2}\left(E_{2}(x)\right)\right\rangle\right)$ and $g_{2}(x)=J_{2}\left(\left\langle x, f_{2}\left(E_{1}(x)\right), g_{2}\left(E_{2}(x)\right)\right\rangle\right)$.

Then

(iv) $f_{1}=f_{2}$, and

(v) $g_{1}=g_{2}$.

Proof: Consider $l$ being a function from $X$ into $\mathbb{N}$ such that for every element $x$ of $X$, if $l(x) \leqslant 0$, then $x \in D$ and if $x \notin D$, then $l\left(E_{1}(x)\right)<l(x)$ and $l\left(E_{2}(x)\right)<l(x)$. Define $\mathcal{P}$ [natural number] $\equiv$ for every element $x$ of $X$ such that $l(x) \leqslant \$_{1}$ holds $f_{1}(x)=f_{2}(x)$ and $g_{1}(x)=g_{2}(x)$. $\mathcal{P}[0]$. For every $k$ such that $\mathcal{P}[k]$ holds $\mathcal{P}[k+1]$ by [3, (13)]. For every natural number 
$k, \mathcal{P}[k]$ from [3, Sch. 2]. For every element $x$ of $X, f_{1}(x)=f_{2}(x)$ and $g_{1}(x)=g_{2}(x)$.

(28) Suppose $\left(E_{1}, E_{2}\right)$ is well founded with minimal set $D$. Then there exists a function $f$ from $X$ into $Y$ such that for every element $x$ of $X$, if $x \in D$, then $f(x)=I(x)$ and if $x \notin D$, then $f(x)=J\left(\left\langle x, f\left(E_{1}(x)\right), f\left(E_{2}(x)\right)\right\rangle\right)$. The theorem is a consequence of $(26)$.

(29) Let us consider functions $f_{1}, f_{2}$ from $X$ into $Y$. Suppose

(i) $\left(E_{1}, E_{2}\right)$ is well founded with minimal set $D$, and

(ii) for every element $x$ of $X$, if $x \in D$, then $f_{1}(x)=I(x)$ and if $x \notin D$, then $f_{1}(x)=J\left(\left\langle x, f_{1}\left(E_{1}(x)\right), f_{1}\left(E_{2}(x)\right)\right\rangle\right)$, and

(iii) for every element $x$ of $X$, if $x \in D$, then $f_{2}(x)=I(x)$ and if $x \notin D$, then $f_{2}(x)=J\left(\left\langle x, f_{2}\left(E_{1}(x)\right), f_{2}\left(E_{2}(x)\right)\right\rangle\right)$.

Then $f_{1}=f_{2}$. Proof: Consider $l$ being a function from $X$ into $\mathbb{N}$ such that for every element $x$ of $X$, if $l(x) \leqslant 0$, then $x \in D$ and if $x \notin D$, then $l\left(E_{1}(x)\right)<l(x)$ and $l\left(E_{2}(x)\right)<l(x)$. Define $\mathcal{P}$ [natural number] $\equiv$ for every element $x$ of $X$ such that $l(x) \leqslant \$_{1}$ holds $f_{1}(x)=f_{2}(x)$. $\mathcal{P}[0]$. For every $k$ such that $\mathcal{P}[k]$ holds $\mathcal{P}[k+1]$ by [3, (13)]. For every natural number $k, \mathcal{P}[k]$ from [3, Sch. 2]. For every element $x$ of $X, f_{1}(x)=f_{2}(x)$.

\section{REFERENCES}

[1] Grzegorz Bancerek. Cardinal numbers. Formalized Mathematics, 1(2):377-382, 1990.

[2] Grzegorz Bancerek. Complete lattices Formalized Mathematics, 2(5):719-725, 1991.

[3] Grzegorz Bancerek. The fundamental properties of natural numbers. Formalized Mathematics, 1(1):41-46, 1990.

[4] Grzegorz Bancerek. The ordinal numbers Formalized Mathematics, 1(1):91-96, 1990.

[5] Grzegorz Bancerek. Bounds in posets and relational substructures. Formalized Mathematics, 6(1):81-91, 1997.

[6] Czesław Byliński. Functions and their basic properties Formalized Mathematics, 1(1): 55-65, 1990.

[7] Czesław Byliński. Functions from a set to a set. Formalized Mathematics, 1(1):153-164, 1990.

[8] Czesław Byliński. Basic functions and operations on functions Formalized Mathematics, 1(1):245-254, 1990.

[9] Czesław Byliński. Partial functions. Formalized Mathematics, 1(2):357-367, 1990.

[10] Czesław Byliński. Some basic properties of sets. Formalized Mathematics, 1(1):47-53, 1990.

[11] Agata Darmochwał. Finite sets Formalized Mathematics, 1(1):165-167, 1990.

[12] B.A. Davey and H.A. Priestley. Introduction to Lattices and Order. Cambridge University Press, 2002.

[13] Marek Dudzicz. Representation theorem for finite distributive lattices Formalized Mathematics, 9(2):261-264, 2001 .

[14] Adam Grabowski. On the category of posets. Formalized Mathematics, 5(4):501-505, 1996.

[15] Kazuhisa Ishida and Yasunari Shidama. Fixpoint theorem for continuous functions on chain-complete posets. Formalized Mathematics, 18(1):47-51, 2010. doi 10.2478/v10037010-0006-x. 
[16] Artur Korniłowicz. Cartesian products of relations and relational structures Formalized Mathematics, 6(1):145-152, 1997.

[17] Andrzej Trybulec. Domains and their Cartesian products Formalized Mathematics, 1(1): $115-122,1990$.

[18] Andrzej Trybulec. Tuples, projections and Cartesian products Formalized Mathematics, 1(1):97-105, 1990.

[19] Wojciech A. Trybulec and Grzegorz Bancerek. Kuratowski - Zorn lemma. Formalized Mathematics, 1(2):387-393, 1990.

[20] Zinaida Trybulec. Properties of subsets. Formalized Mathematics, 1(1):67-71, 1990.

[21] Glynn Winskel. The Formal Semantics of Programming Languages. The MIT Press, 1993.

[22] Edmund Woronowicz. Relations and their basic properties. Formalized Mathematics, 1 (1):73-83, 1990.

[23] Edmund Woronowicz. Relations defined on sets, Formalized Mathematics, 1(1):181-186, 1990.

[24] Mariusz Żynel and Czesław Byliński. Properties of relational structures, posets, lattices and maps Formalized Mathematics, 6(1):123-130, 1997.

Received February 11, 2014 